\title{
Comparison of Pulsatile Pressure Flow through a Specific Patient's Rigid and Deformable Arteriovenous Fistula: In Vitro Study
}

\author{
J. F. Rangel ${ }^{1}$, W. B. A. Santos ${ }^{1}$, L. H. Pinheiro ${ }^{1}$, T. H. C. Costa ${ }^{1}$ \\ K. L. Bessa ${ }^{1 \dagger}$ and J. P. Ortiz ${ }^{2}$ \\ ${ }^{1}$ Federal University of Rio Grande do Norte, Natal, Rio Grande do Norte, 59078-970, Brazil \\ ${ }^{2}$ University of São Paulo, São Paulo, São Paulo, 05508-030, Brazil \\ †Corresponding Author Email: klbessa@ct.ufrn.br
}

(Received September 7, 2020; accepted May 3, 2021)

\begin{abstract}
Arteriovenous fistula (AVF) is the most widely used vascular access by patients undergoing hemodialysis, however, even though the most widely used access to AVF has a high failure rate, and can be affected by problems during its use, among the most common highlights intimal hyperplasia, thrombosis and stenosis. Studies suggest that recurrent problems in this type of access are directly linked to geometry, flow conditions and stiffness of the vascular wall by the vessels that compose it. The present work seeks to analyze the variation of pressure and flow in rigid and flexible AVF models manufactured from data from an actual patient undergoing treatment. The study was carried out from the acquisition and processing of the patient's medical examinations (computed tomography), the creation of the geometry, treatment and modeling of said patient, the manufacturing of the AVF models by 3D printing and injection in mold, experimental analysis with pulsatile flow conditions, close to the real physiological conditions, and data analysis. The results obtained show the influence of vascular wall stiffness on flow conditions. In the rigid and flexible model, pressure peaks ranged from $170.98 \mathrm{mmHg}$ to $172.44 \mathrm{mmHg}( \pm 0.02)$ and $69.83 \mathrm{mmHg}$ to $116.63 \mathrm{mmHg}( \pm 0.03)$, respectively. The pressure drop between entry and exit in the AVF was also analyzed, presenting a greater value in the flexible model, being approximately three times that of the rigid model. The observed results show the direct relation of the deformation in the flow conditions in the system, and consequently, its direct influence on the pathologies of the vascular system, especially the AVF.
\end{abstract}

Keywords: Arteriovenous fistula; Rigid; Deformation; Pressure variation; Flow variation.

\section{NOMENCLATURE}

$\begin{array}{llll}\text { AVF } & \text { Arteriovenous Fistula } & \mathrm{C} & \text { compliance } \\ \text { BSN } & \text { Brazilian Society of Nephrology } & \mathrm{d}_{\mathrm{s}} & \text { diameter in the systole } \\ \text { CKD } & \text { Chronic Kidney Disease } & \mathrm{d}_{\mathrm{d}} & \text { diameter in diastole } \\ \text { ESRD } & \text { End-Stage Renal Disease } & \mathrm{P}_{\mathrm{s}} & \text { systolic pressure } \\ \mathrm{RRT} & \text { Renal Replacement Therapy } & \mathrm{P}_{\mathrm{d}} & \text { diastolic pressure } \\ \mathrm{m} & \text { meter } & \mathrm{t} & \text { time } \\ \mathrm{N} & \text { Newton } & \mathrm{T} & \text { time normalization factor } \\ \mathrm{N} / \mathrm{m}^{2} & \text { Pascal }(\mathrm{Pa}) & \mathrm{t} / \mathrm{T} & \text { normalized time } \\ \rho & \text { density } & \mathrm{VA} & \text { Vascular Access } \\ \mu & \text { dynamic viscosity } & \mathrm{IH} & \text { Intimal Hyperplasia }\end{array}$

\section{INTRODUCTION}

Chronic kidney disease (CKD) is a disease characterized by a slow, progressive and irreversible decrease in renal functions. In its terminal stage, called end-stage renal disease (ESRD), the patient

needs therapeutic treatment aimed at remedying lost kidney functions. There are two alternatives for the treatment of ESRD, which are called Renal Replacement Therapy (RRT): kidney transplantation and dialysis (Webster et al. 2017). Mihajlovic et al. (2017) estimated that almost $10 \%$ of the world's 
population is affected by CKD. According to the Brazilian Society of Nephrology (BSN), each year there is an increase in the number of patients in dialytic treatment, from 42 thousand in 2000 to more than 133 thousand patients in 2018, an increase of $212 \%$ in recent years (Thomé et al. 2018). Arteriovenous fistula (AVF) is the ideal form of vascular access (VA) used by patients with CKD in hemodialysis treatment. The AVF is the preferred vascular access because it has lower patency rates and fewer complications when compared with arteriovenous grafts and catheters (Astor et al. 2005; Wasse 2008; Besarab 2008; Akoh 2009; Briones et al. 2010). Even though it is a well-recommended access, some problems may render it useless, such as, pseudoaneurysms, intimal hyperplasia (IH), stenosis, among others. Remuzzi and Bozzetto (2017) point out that about $60 \%$ of AVF do not reach the ideal state of use after their conception. AVF consist of the surgical connection of an artery and vein, which undergo remodeling because of the short pressure circuit caused by the union of these vessels. Geometric remodeling and the new inherent flow conditions are the factors associated with possible pathophysiological sites and, consequently, problems in vascular access. After the creation of the $\mathrm{AVF}$, there is a variation in the pressure field, this change being one of the important parameters regarding maturation and pathological conditions of access. We can recognize the pressure drop caused by flow instabilities in the anastomosis as being a global factor linked to the hemodynamics of vascular access (Botti et al. 2013). The correct analysis of pressure variation in the VA helps in predicting vascular remodeling in the short term, and in the ability to maintain the patency of access in the long term, thus being a global parameter for analyzing access conditions, avoiding unnecessary surgical interventions. According to Browne et al. (2015) and Pylayeva-Gupta (2011), fluctuations in speed and pressure, in addition to the high shear stress on vascular walls, are also parameters that directly influence the external remodeling of the vein. In addition to external remodeling, the influence of high pressure on the thickening of the venous wall is highlighted (Botti et al. 2013). The aim of this study is to compare pressure pulses at specific sites of the arteriovenous fistula in rigid and flexible models built from real data from a patient.

\section{METHODOLOGY}

\subsection{Fistula Models}

The vascular geometry of a real patient with brachiocephalic AVF was reconstructed threedimensionally, using DICOM files (Digital Imaging and Communications in Medicine) from computational tomography, with the aid of the InVesalius software (CTI, Campinas, Brazil). The structure of the vascular wall was modeled from an offset of $3 \mathrm{~mm}$, in addition to an increase in the length of entry (proximal artery) and exit (proximal vein) for connection to the workbench and design of access points for acquisition pressure, as shown in Fig. 1.

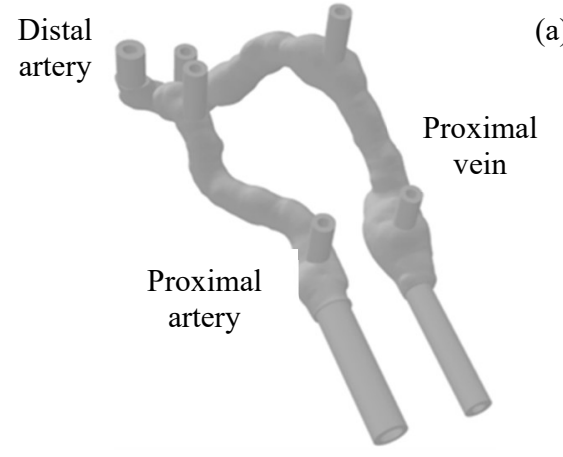

(a)

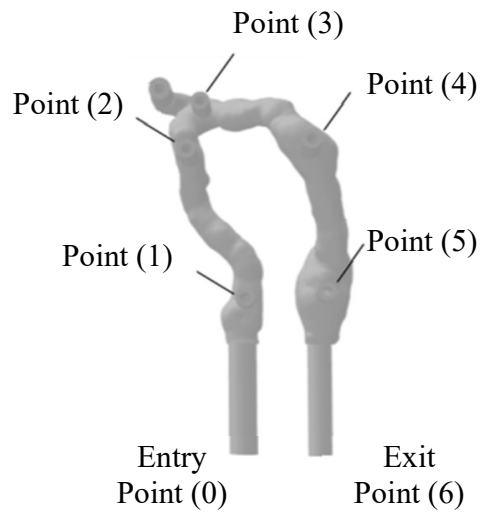

(b)

Fig. 1. Arteriovenous fistula: (a) sections and; (b) analyses points.

The approximate values of the diameters of the entry, exit, points (1), (2), (4) and (5) are, respectively, 6 $\mathrm{mm}, 6 \mathrm{~mm}, 7.13 \mathrm{~mm}, 14.06 \mathrm{~mm}$ and $16.52 \mathrm{~mm}$. Point (5), site of the anastomosis, does not have a cross-sectional area close to uniformity, therefore, we cannot define an approximate diameter, being, having an area of $159.53 \mathrm{~mm}^{2}$.

The linear distances between the entrance and the point (1), point (1) and point (2), point (4) and point (5) and between point (5) and exit are $75 \mathrm{~mm}$. Between point (2) and point (3) and point (3) and point (4) is $25 \mathrm{~mm}$, adding a total distance between entry and exit of $350 \mathrm{~mm}$.

The thickness value of the flexible AVF model was $2 \mathrm{~mm}$, constant throughout the system, and in the rigid model the thickness does not have a great influence on the flow since the walls do not deform, thus having a thickness of $3 \mathrm{~mm}$ ensuring a total rigidity of the system.

After computational modeling, the manufacturing processes for the two types of AVF were performed. The rigid AVF was built using rapid prototyping (3D printing) by fusion deposition modeling (FDM) using the VOID 3D 1+ printer model (VOID 3D, Digital Metropolis Institute, Brazil), with $0.2 \mathrm{~mm}$ resolution, in ABS (acrylonitrile butadiene styrene) and $100 \%$ of filled. The flexible AVF was manufactured using injection mold. The mold and the core were modeled and manufactured using 3D printing, these elements were designed in order to guarantee the structural uniformity of the AVF and 
good surface quality (Fig. 2). The printer model and parameters used are the same as the rigid AVF.

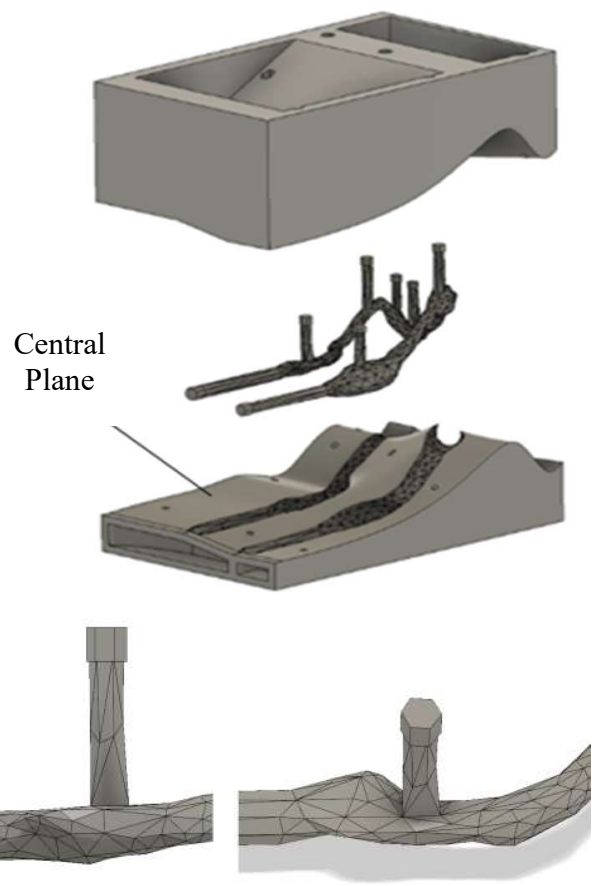

Fig. 2. Mold and access points - virtual model.

\subsection{Flow conditions}

The experimental bench was designed to simulate the physiological pulsatile flow, presenting the systolic and diastolic phases of the pulses, associated with the functions predefined in the program. The control system sends an A/D (analog / digital) pulse signal (Fig. 3) to the pump motor, controlling its rotation. The implemented $\mathrm{A} / \mathrm{D}$ pulse is based on 4 different mathematical functions, developed based on the pulse present in the work developed by Sivanesan et al. (1999), which together characterize the general function. The functions that make up the pulse can be seen in Fig. 3.

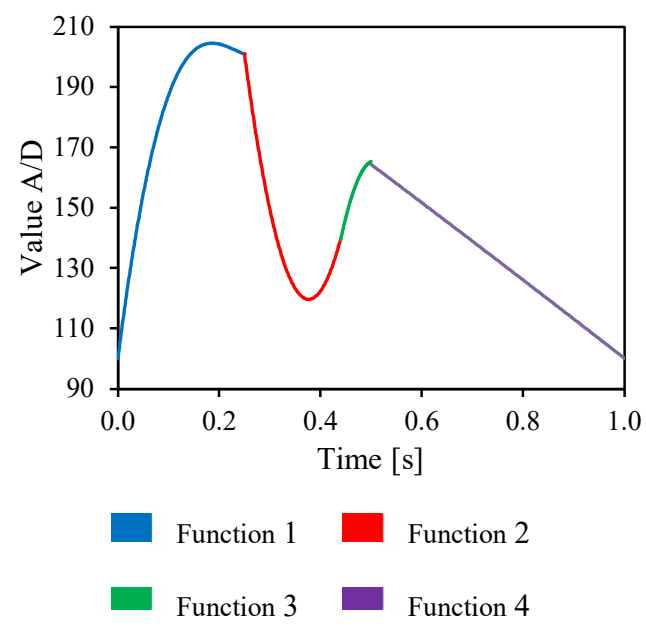

Fig. 3. Control function - Diaphragm pump.
The analysis was performed with the distal artery occluded, in order to guarantee the flow from the proximal artery to the vein. This condition represents reality, since most flows in AVF occur in this way, from a region of high pressure (arterial system) to a region of low pressure (venous system).

\subsection{Flow circuit}

The experiments were carried out on a bench (Fig. 4) developed to simulate the blood pressure pulse, which consists of a pumping system using a diaphragm pump coupled to an engine CF2201a. The variation of the load applied to the motor was promoted by a power circuit, through a transistor TIP 120 , and this was driven by a control circuit using the modulation width pulse technique. Globe valves were used to increase the resistance in the model. The pressure profile obtained showed a systolic elevation with a marked behavior, followed by a slow elevation to the systolic peak with a pressure of approximately $16 \mathrm{kPa}$, incisure, slow diastolic decline and diastolic pressure of approximately $12 \mathrm{kPa}$. The fluid used was water at $30{ }^{\circ} \mathrm{C}$ with a specific mass of $\rho=996 \mathrm{~kg} / \mathrm{m}^{3}$ and dynamic viscosity of $\mu=0.798 \mathrm{E}-3 \mathrm{Ns} / \mathrm{m}^{2}$. The fluid was pumped in a closed path, through a pipeline, from a reservoir to the in vitro model.

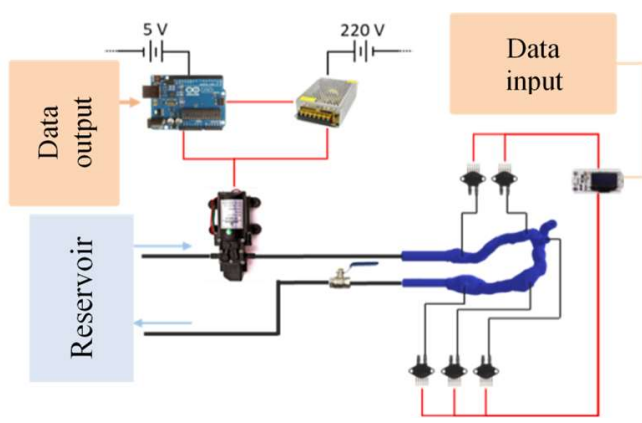

Fig. 4. Experimental bench (flow).

\subsection{Deformation circuit}

Deformation analysis was performed using a video capture system attached to the pulsating flow bench (Fig. 4) as shown in Fig. 5. The system consisted of a camera with a resolution of $1920 \times 1080$ pixels and a capture rate of $60 \mathrm{fps}$ and height-adjustable support.

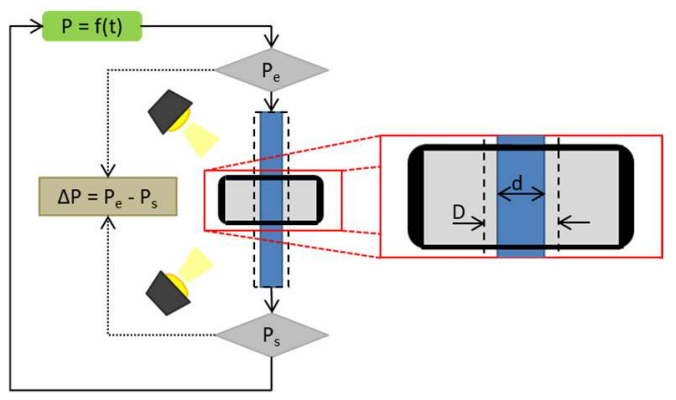

Fig. 5. Experimental bench (deformation). 
Through the deformation data, it was possible to analyze the compliance of both systems based on the methodology developed by Reneman et al. (1986).

$C=\frac{d_{s}-d_{d}}{2\left(P_{s}-P_{d}\right)} \cdot \pi d_{d}$

Where, $\mathrm{C}\left(\mathrm{N} / \mathrm{m}^{4}\right)$ is compliance, $\mathrm{d}_{\mathrm{s}}(\mathrm{m})$ the diameter in the systole, $\mathrm{d}_{\mathrm{d}}(\mathrm{m})$ the diameter in diastole, $\mathrm{P}_{\mathrm{s}}(\mathrm{Pa})$ the systolic pressure and $\mathrm{P}_{\mathrm{d}}(\mathrm{Pa})$ the diastolic pressure.

\subsection{In vitro Measurements}

For signal acquisition, pressure transducers MPX5050DP with the capacity to measure manometric pressures up to $50 \mathrm{kPa}$ were used. An analog-to-digital signal converter 15 bits ADS1115 was installed, which provided an increase in accuracy in the measured pressure from $48.82 \mathrm{~Pa}$ to $1.52 \mathrm{~Pa}$. A low-pass filter was installed to attenuate the noise effects inherent in the crystal pressure transducer. The flow was measured by a flow sensor YFS401 by counting revolutions promoted by a hall sensor and a magnetic body coupled to a turbine.

The codes, both for the control of the engine and for the acquisition of pressure and flow data, were programmed with $\mathrm{c} / \mathrm{c}++$, compiled through Arduino's Integrated Development Environment and implemented in microcontrollers ESP32 and Arduino Uno.

\subsection{Experimental procedure: Flow analsyses}

From the implementation of pulsatile flow in the system, pressure values were obtained at predetermined points during the systolic and diastolic phases of the cycle, as a function of time.

Points (1), (2), (3), (4) and (5) were arranged in the upper plane of the AVF, with two points located in the arterial segment, one point in the central region of the anastomosis and two points in pseudoaneurysms located in the venous segment. The pressure was also analyzed at the points of entry (point (0)) and exit (point (6)) of the AVF, in order to study the pressure drop in the two different models.

\section{RESULTS AND DISCUSSION}

\subsection{AVF Model}

\subsubsection{Rigid AVF}

The rigid AVF was printed in $3 \mathrm{D}$, with an approximate printing time of $24 \mathrm{hrs}$. After it was manufactured, preliminary tests were carried out observing micro leaks in the printed AVF, which were remedied through a correction treatment and external surface improvement with the application of acetone vapor (concentration: 98\%). The result of the manufacturing of the rigid AVF can be seen in Fig. 5.

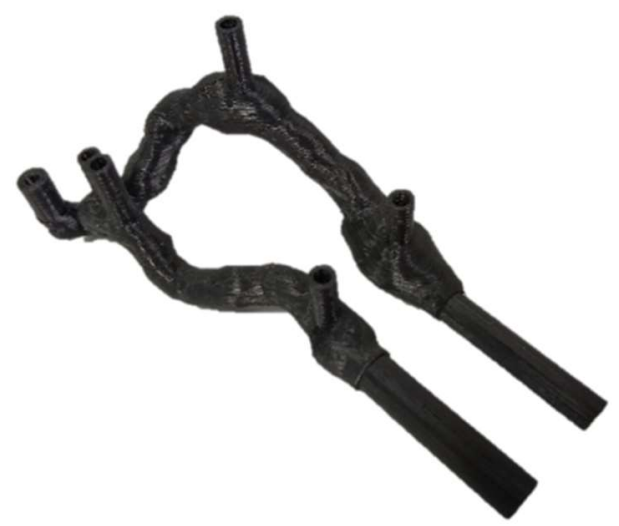

Fig. 6. AVF rigid model.

\subsubsection{Deformable AVF}

The mold manufacturing method was developed after initial attempts at digital painting manufacturing were unsuccessful. The manufacturing process for flexible AVF by painting was resulted in AVF with non-uniform thickness and the presence of bubbles superficially and internally.

Some attempts to manufacture the mold were unsuccessful, presenting problems, among which the following stand out: centralization of the parts of the mold and the core, difficulty in removing the AVF after curing and fixing the mold. The parts of the final mold manufactured were fixed by means of screws, centered on the plane formed in the centerline of the access (Fig. 2), facilitating the unmolding of the AVF after curing. The core initially used was made of paraffin; however, the injection of silicone caused the breakage of this element, and, consequently, a failure in manufacturing. In a second attempt, the core made by printing $3 \mathrm{D}$ in $\mathrm{ABS}$ was used, which is more resistant to silicone injection, and which, after curing, was removed by dissolving it in acetone. The centralization of this element (core) was done through the extremities (distal artery, distal vein and proximal artery), ensuring uniformity in the thickness of the vascular wall. Fig. 6 shows the mold used to manufacture the flexible AVF.

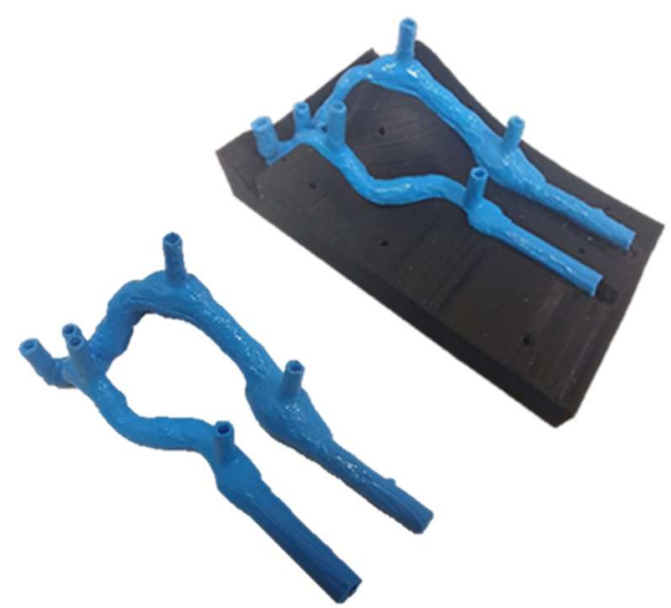

Fig. 7. AVF flexible model 


\subsection{Flow analysis}

In this section we present the values for pressure variation, pressure drop and flow variation in the vascular system in the two AVF models submitted to pulsatile flow.

\subsubsection{Pressure variation}

The pressure values observed, as a function of normalized time, in both AVF models are shown for points 0 (AVF entry), 1, 2, 3, 4, 5, and 6 (AVF exit), according to Fig. 1, in Figs. 7 to 11, respectively.

At point 0 (AVF entry - Fig. 7), the first differences between the pressure pulse were observed comparing the two AVF models. When the results are related, there is an amortization in the pulse in the flexible model and a lower peak value when compared to the pressure pulse in the rigid model. In Fig. 7 the pressure pulses at the entrance of the AVF in both models show a significant difference, where the maximum values observed were $166.44 \mathrm{mmHg}$ and $101.56 \mathrm{mmHg}$ for the rigid and flexible AVF, respectively. It is observed that the pressure pulse does not have a well-defined systolic and diastolic phase in the flexible model, a fact attributed to a local change in the rigidity of the conduits.

At point 1, a point located in the arterial region immediately after the entrance of the AVF, higher values were observed in the pressure peaks comparing the entrance of the AVF, in both models. These higher pressure values are due to a curvature in the AVF geometry; the peak values, as observed in Fig. 8 were for the rigid and flexible model, respectively, $172.44 \mathrm{mmHg}$ and $108.46 \mathrm{mmHg}$. The pulses also have more organized flow phases.

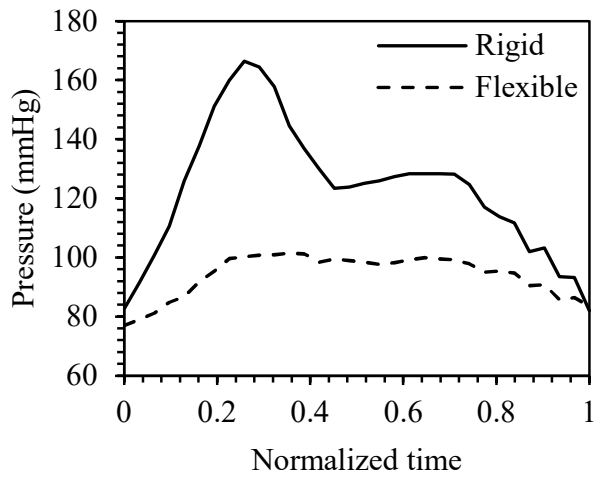

Fig. 8. Pressure drop vs. normalized time: Entry (Point 0).

At point 2, the second point in the arterial segment, located in a higher region in relation to point 1 and the entry, the pressure values observed were lower, in the two AVF models, when compared to the values of the previous points, with the following peak values: $155.64 \mathrm{mmHg}$ and $101.41 \mathrm{mmHg}$ (Fig. 9).

Point 3, located in the upper plane of the anastomosis region (union of the venous and arterial segments), presented, in both cases, the lowest values for pressure pulses and peak values (rigid AVF: 150.98 mmHg; flexible AVF: $99.53 \mathrm{mmHg}$ ), as shown in Fig. 10. These results were expected, given that this region has adverse pressure values, with high values (stagnation region) and low values (recirculation regions) of pressure in different places.

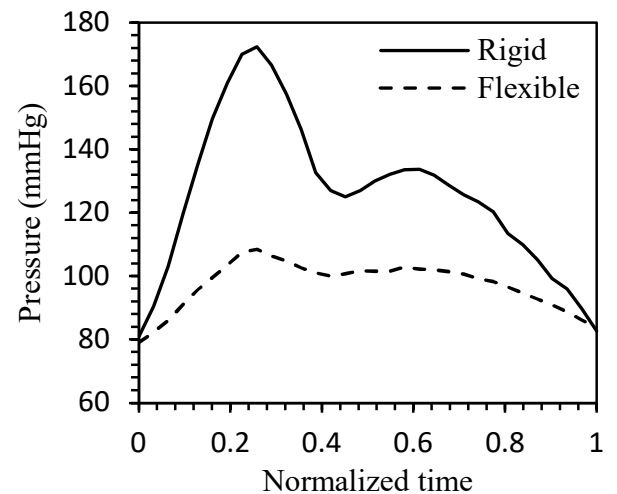

Fig. 9. Pressure drop vs. normalized time: Point 1.

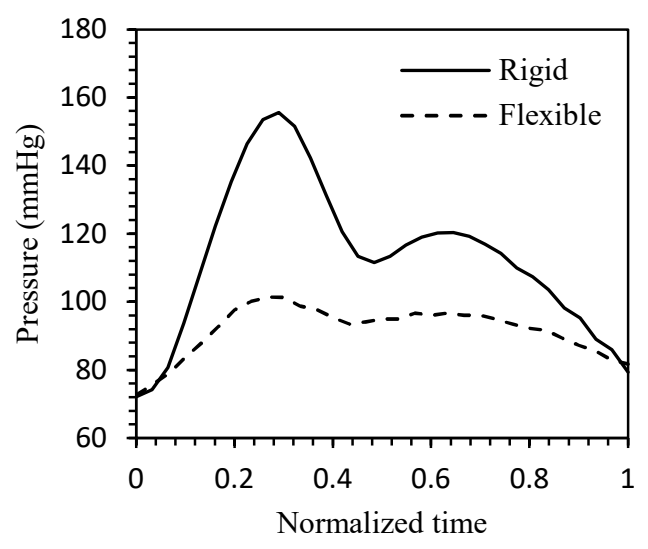

Fig. 10. Pressure drop vs. normalized time: Point 2.

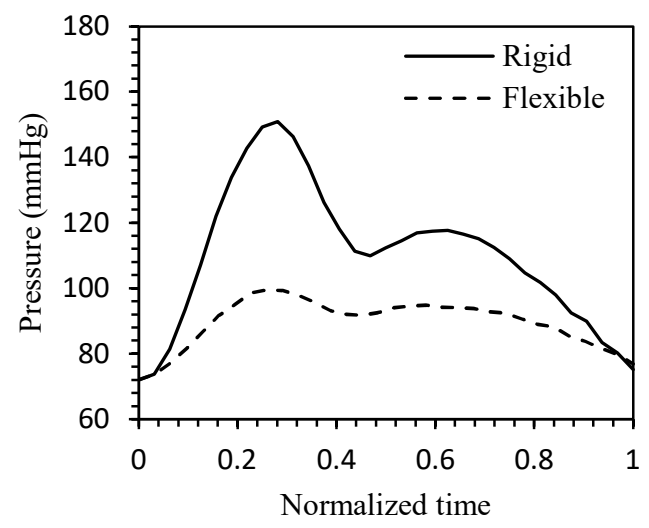

Fig. 11. Pressure drop vs. normalized time: Point 3

Points 4 and 5, located in the AVF venous region in pseudoaneurysms resulting from the punctures, showed higher pulses and pressure peaks in the system due to the increase in the cross-sectional area (Fig. 11). This occurs based on the principle of 
energy conservation of the system, showing that with the increase in the cross-sectional area there is a decrease in velocity at that point in the system and as a consequence a local increase in pressure.
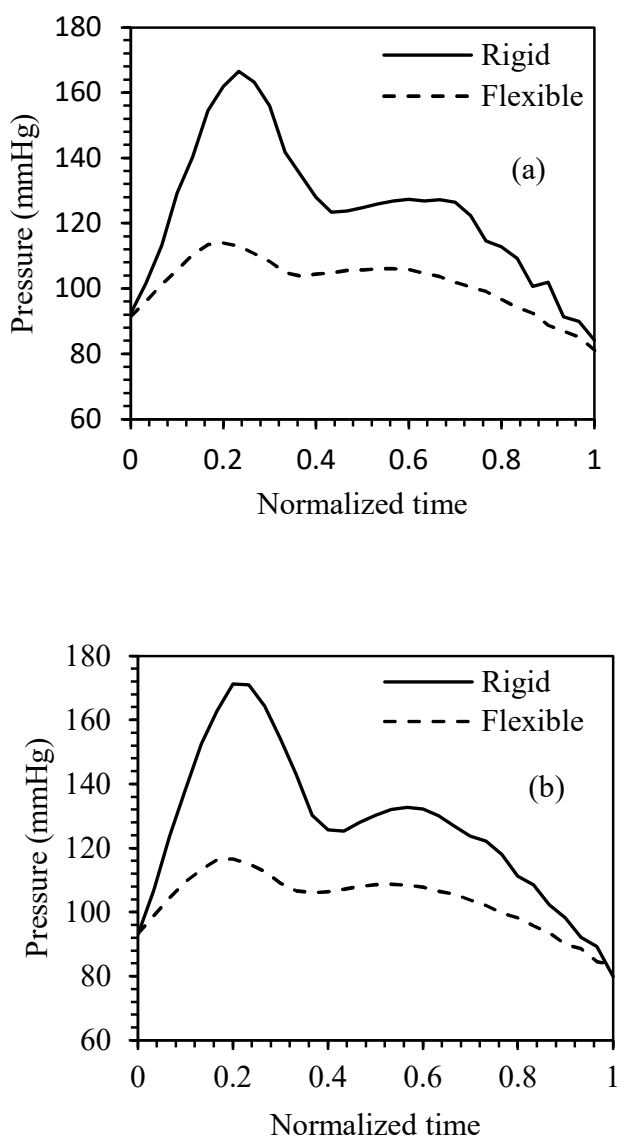

Fig. 12. Pressure drop vs. normalized time: a) Point 4; b) Point 5.

At the exit of the AVF (Point 6 - Fig. 01) there was a significant drop in the local pressure value of the flexible model when compared to the value in the rigid model (Fig. 12), in addition to a greater drop in the pressure value, comparing entry and exit of the $\mathrm{AVF}$, in the flexible model. This pressure drop is directly associated with the energy loss resulting from the deformation of the system. The peak values observed in the pressure pulses of both rigid and flexible models at that point were, respectively, $155.71 \mathrm{mmHg}$ and $69.83 \mathrm{mmHg}$.

In the rigid AVF model, as seen earlier, higher values for pressure peaks and high pulse increase rates are observed, reaching elevated pressure values in a short period of time. The high rigidity of the model's wall has a direct influence on the results obtained, given that the values of pressure entering the system remain integral with respect to the energy loss caused by the deformation of the wall. Table 1 shows the peak pressure values at the points of the rigid AVF, and the respective times of the pulse phases.

The pressure peaks in the rigid AVF occurred at the average normalized time of $\mathrm{t} / \mathrm{T}=0.258 \pm 0.03$ and the systolic period at the average time of $\mathrm{t} / \mathrm{T}=0$ to
$0.468 \pm 0.01$, showing the uniformity of the results in the rigid model with respect to the time when these phenomena occur. The highest pressure values were observed at points (1) and (5), located just after the entrance and before the exit, respectively. The region of point (1) has a chaotic flow contributing to the elevation of pressure locally in the system, whereas in point (5), a region of pseudoaneurysms, the elevation of pressure occurs due to the increase in area.

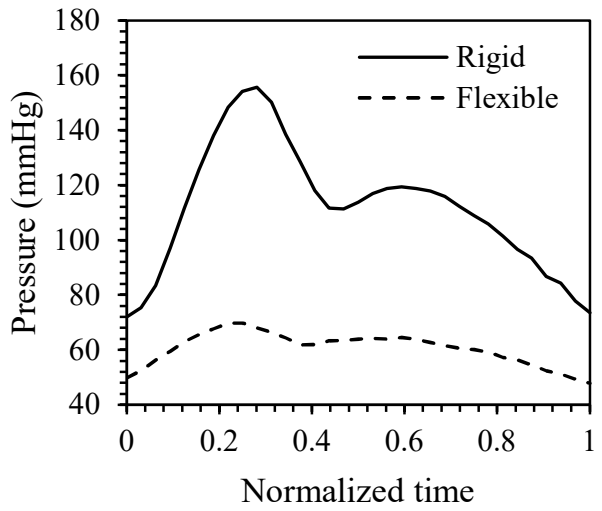

Fig. 13. Pressure drop vs. normalized time: Exit (Point 6)

Table 1 Peak pressure, normalized time (peak) and systolic period of the pulse: rigid AVF.

\begin{tabular}{|l|c|c|c|}
\cline { 2 - 4 } \multicolumn{1}{c|}{} & \multirow{2}{*}{\begin{tabular}{c} 
Peak \\
pressure \\
\cline { 3 - 4 } \multicolumn{1}{c|}{}
\end{tabular}} & \multicolumn{2}{|c|}{ Normalized time (t/T) } \\
\cline { 3 - 4 } \multicolumn{1}{c|}{} & 166.44 & 0.258 & $0-0.484$ \\
\hline Entry & 155.71 & 0.281 & $0-0.468$ \\
\hline Exit & 172.44 & 0.258 & $0-0.451$ \\
\hline Point (1) & peak) & (systole) \\
\hline Point (2) & 155.64 & 0.290 & $0-0.483$ \\
\hline Point (3) & 150.98 & 0.281 & $0-0.469$ \\
\hline Point (4) & 166.51 & 0.233 & $0-0.467$ \\
\hline Point (5) & 170.94 & 0.200 & $0-0.433$ \\
\hline
\end{tabular}

The lowest pressure value in the rigid model is observed in the anastomosis region, a region susceptible to recirculation, and as a result there is a drop in local pressure, as stated by Botti et al. (2013). There is great importance in the flow analysis in this region, which can suffer from pathologies due to the inherent flow conditions, Cunnane et al. (2017) highlights this region as prone to the formation of hyperplasia and deposition of cellular material (atherosclerosis).

Lower pulses and pressure spikes were observed in the flexible AVF model, as seen previously. This decrease in pulse values shows a drop in the energy of the system, related to the deformation of the vascular wall, working directly as a flow damping mechanism, where part of the fluid energy that causes the deformation is "dissipated" and part is returned to the flow. 
Table 2 shows the peak values and the systolic pulse period in the flexible AVF model.

Table 2 Peak pressure, normalized time (peak) and systolic period of the pulse: deformable AVF.

\begin{tabular}{|c|c|c|c|}
\hline & \multirow{2}{*}{$\begin{array}{c}\text { Peak } \\
\text { pressure } \\
(\mathrm{mmHg})\end{array}$} & \multicolumn{2}{|c|}{ Normalized time $(t / T)$} \\
\hline & & (peak) & (systole) \\
\hline Entry & 101.56 & 0.354 & $0-0.548$ \\
\hline Exit & 69.83 & 0.250 & $0-0.343$ \\
\hline Point (1) & 108.46 & 0.258 & $0-0.419$ \\
\hline Point (2) & 101.41 & 0.266 & $0-0.433$ \\
\hline Point (3) & 99.53 & 0.258 & $0-0.452$ \\
\hline Point (4) & 114.09 & 0.200 & $0-0.300$ \\
\hline Point (5) & 116.63 & 0.200 & $0-0.366$ \\
\hline
\end{tabular}

Peak values occurred at an average normalized time of $\mathrm{t} / \mathrm{T}=0.258 \pm 0.05$ and the systolic period between an average time of $\mathrm{t} / \mathrm{T}=0$ to $0.419 \pm 0.08$, showing small variations, related to the influence of deformation, and, consequently, the longer time needed to reach peak pressure (slower increase rates). The highest values at peak pressure occur at points (4) and (5), located in the venous region at pseudoaneurysms.

The deformation of the AVF wall is one of the most important parameters with regard to the flow conditions in the access. During the pulsatile cycle the pressure values fluctuate so that the loading imposed on the wall of the AVF is alternated. Thus, the deformation is more accentuated at certain periods of the pulse, and the energy, analyzed in the form of pressure, is decreased by the deformation of the system, which was evident from the analysis of the results.

As Botti et al. (2013) shows, the remodeling of the vascular structure, the thickening of the access walls, and the increased rigidity of the AVF walls, are caused by the non-physiological variation of the pulse. Consequently, due to the increased stiffness of the vascular wall, among other factors, a direct increase in the global value of the system pressure is observed, thus causing an increase in the incidence of problems in the AVF in the short and long term.

\subsubsection{Compliancce}

Through the deformation experimental data in the flexible AVF model, in a pulsatile flow regime, the compliance of some sections of the system was analyzed. Figure 14 shows the compliance graph of points (1), (2), (4) and (5).

Based on Eq. (1) it is observed that, for any pressure value, the compliance in the rigid AVF model is zero $(0)$, since there is no variation in the section due to the rigidity of this access model.

The values for compliance, observed in the flexible
AVF, ranged from 2,183E-09 $\mathrm{m} 4 / \mathrm{N}$ to $8,835 \mathrm{E}-09$ $\mathrm{m} 4 / \mathrm{N}$. This difference in the compliance values between the two AVF models mainly shows that the greater stiffness of the arterial wall causes a decrease, or in our case a null value, of variation in the internal volume with increased pressure. This non-variation in volume mainly implies a decrease in the capacity of the venous region to serve as a "reserve" if the blood volume that will be supplied to the cardiovascular system when necessary.

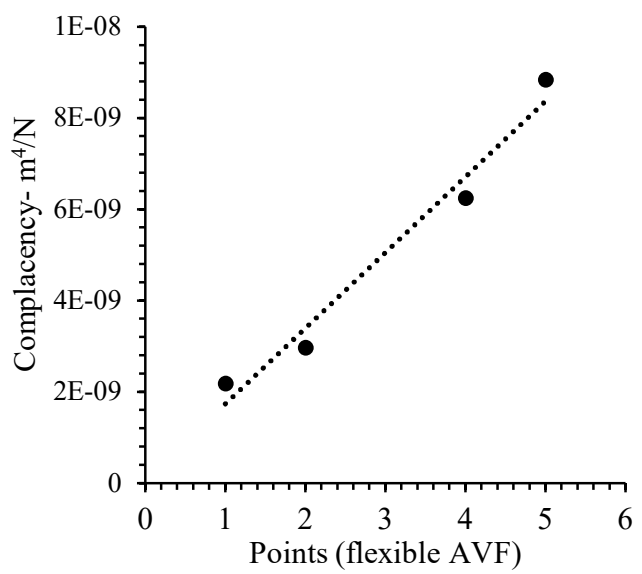

Fig. 14. Compliance - flexible AVF

\subsubsection{Flow variation}

The flow pulses acquired in the rigid and flexible AVF are shown in Fig. 14. Higher flow values, average and peak, were observed in the rigid AVF models. The mean values were $1360.18 \mathrm{ml} / \mathrm{min}$ and $1335.87 \mathrm{ml} / \mathrm{min}$ in the rigid and deformable AVF models, respectively, showing a difference of 24.31 $\mathrm{ml} / \mathrm{min}$. The flow at the peak of the pulse was $1660.62 \mathrm{ml} / \mathrm{min}$ in the rigid AVF and $1409.3 \mathrm{ml} /$ min in the flexible AVF, observing a difference of $251.32 \mathrm{ml} / \mathrm{min}$.

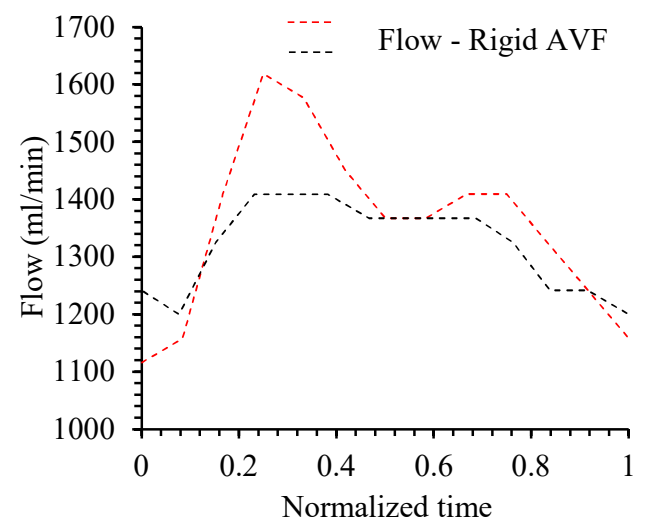

Fig. 15. Flow pulse in rigid and flexible AVF.

The decrease in flow values between the pulses of the rigid and flexible AVF model is the result of system deformation and initial accumulation of a portion of the fluid volume. 


\subsubsection{Pressure drop}

The pressure pulses at the entrance and exit of the AVF were obtained, and the pressure loss was analyzed in both models, observing a greater pressure drop in the flexible AVF model. These results are corroborated by the graph of Fig. 16. The value of the average pressure drop observed in the system was $9.175 \mathrm{kPa}$ in the flexible AVF and 8.938 $\mathrm{kPa}$ in the rigid $\mathrm{AVF}$, and a pressure drop in the peak pulse in the rigid and flexible models of $12.43 \mathrm{kPa}$ and $11.48 \mathrm{kPa}$, respectively.

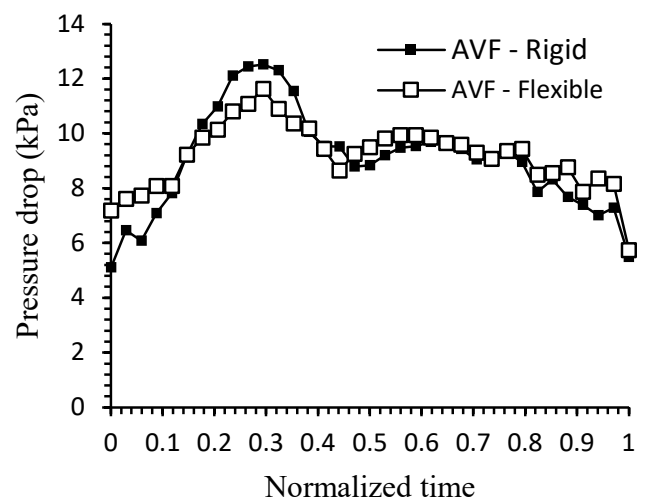

Fig. 16. Pressure drop in rigid and flexible models.

It is observed that a lower stiffness of the AVF wall induces a greater drop in mean in the system, evidencing, as previously stated, that part of the flow energy is converted into deformation of the access wall

\section{Conclusion}

It was observed in the flexible AVF model a greater drop in the flow and pressure pulses when compared to the rigid model, showing, among other aspects, that the stiffness of the arterial wall has a direct influence on the flow requirements on it. This shows that in vessels with greater vascular wall stiffness the predominance of problems related to high pressure and greater shear stress on the wall, such as intimal hyperplasia.

Thus, knowing the phenomena caused by the deformation of the system contributes greatly to the design and development of biomedical devices, improving the adaptation to physiological conditions of the systems in question, along with helping to monitor the real physiological conditions of patients who are in treatment or carriers of diseases.

\section{ACKNOWLEDGEMENTS}

I would like to express my thanks to the financial support of the Coordenação de Aperfeiçoamento de Pessoal de Nível Superior - Brasil (CAPES).

\section{REFERENCES}

Astor, B.C., J. Eustace, N. R. Powe, M. J. Klag, N.
E. Fink and J. Coresh (2005). Type of vascular access and survival among incident hemodialysis patients: the choices for healthy outcomes in caring for ESRD (choice) study. Journal of the American Society of Nephrology 16(5), 1449-1455.

Akoh, J. A. (2009). Prosthetic arteriovenous grafts for hemodialysis. Journal of Vascular Access 10(3), 137-147.

Besarab, A. (2008). Resolved: Fistulas are preferred to grafts as initial vascular access for dialysis. Pro. Journal of the American Society of Nephrology 19(9), 1629-1631.

Botti, L., K. Van Canneyt, R. Kaminsky, T. Claessens, R. N. Planken, P. Verdonck, A. Remuzzi and L. Antiga (2013). Numerical Evaluation and Experimental Validation of Pressure Drops Across a Patient-Specific Model of Vascular Access for Hemodialysis. Cardiovascular Engineering and Technology 4(4), 485-499.

Briones, L., A. Diaz Moreno, S. Sierre, L. Lopez, J. Lipsich and M. Adragna (2010). Permanent vascular access survival in children on longterm chronic hemodialysis. Pediatric Nephrology 25(9), 1731-1738.

Browne, L. D., P. Griffin, K. Bashar, S. R. Walsh, E. G. Kavanagh and M. T. Walsh (2015). In Vivo Validation of the In Silico Predicted Pressure Drop Across an Arteriovenous Fistula. Annals of Biomedical Engineering 43(6), 1275-1286.

Cunnane, C. V., E. M. Cunnane and M. T. Walsh (2017). A Review of the Hemodynamic Factors Believed to Contribute to Vascular Access Dysfunction. Cardiovascular Engineering and Technology 8(3), 280-294.

Mihajlovic, M., L. P. van den Heuvel, J. G. Hoenderop, J. Jansen, M. J. Wilmer, A. F. Westheim, W. A. Allebes, D. Stamatialis, L. B. Hilbrands and R. Masereeuw (2017). Allostimulatory capacity of conditionally immortalized proximal tubule cell lines for bioartificial kidney application. Scientific Reports 7(1), 1-14.

Pylayeva-Gupta, Y. A. (2011). Longitudinal Study of Remodeling in a Revised Peripheral Artery Bypass Graft Using 3D Ultrasound Imaging and Computational Hemodynamics. Journal of Biomechanical Engineering 23(1), 1-7.

Remuzzi, A. and M. Bozzetto (2017). Biological and physical factors involved in the maturation of arteriovenous fistula for hemodialysis. Cardiovascular Engineering and Technology 8(3), 273-279.

Reneman, R. S., T. van Merode, P. Hick, A. M. M. Muytjens and A. P. G. Hoeks (1986). Agerelated changes in carotid artery wall properties in man. Ultrasound Med. Biol. $12,465-$ 471.

Sivanesan, S., T. V. How, R. A. Black and A. Bakran 
J. F. Rangel et al. / JAFM, Vol. 14, No. 6, pp. 1593-1601, 2021.

(1999) Flow patterns in the radiocephalic arteriovenous fistula: An in vitro study. Journal of Biomechanics 32(9), 915-925.

Thomé, F. S., R.C. Sesso, A. A. Lopes, J. R. Lugon and C. T. Martins (2018) Inquérito Brasileiro de Diálise Crônica. Brazilian Journal of Nephrology 41(2), 208-214.
Wasse, H. (2008). Reducing tunneled hemodialysis catheter morbidity catheter-related mortality among ESRD patients. Seminars in Dialysis 21(6), 547-549.

Webster, A. C., E. V. Nagler, R. L. Morton, and P. Masson (2017). Chronic Kidney Disease. The Lancet 389, 1238-1252. 